\section{Foundational strategies in PD therapeutics}

\section{By Michael J. Haas, Senior Writer}

The Michael J. Fox Foundation for Parkinson's Research has been playing at all stages of the development spectrum. Most recently it has granted \$2.4 million to fund research into repurposing marketed drugs to treat Parkinson's disease and is collaborating on the development of a new mouse model for the indication that will be freely available to researchers.

According to CEO Todd Sherer, "A key aspect of what we do is de-risking Parkinson's disease research by placing bets on ideas and therapies" that face scientific or business obstacles. By funding research to overcome these obstacles, he said MJFF expects to make $\mathrm{PD}$ a more attractive investment to pharmas and venture capitalists.

The six grants awarded under MJFF's new Repositioning Drugs for PD 2011 program are aimed at generating data to demonstrate that existing products could have a therapeutic benefit in PD, thereby encouraging companies to repurpose those products, he said.

Five projects are in preclinical testing, while the sixth is in the clinic. The products being tested range from a major depression drug to a generic tuberculosis (TB) vaccine, said Nate Herpich, MJFF's associate director of research communications.

One grant went to a team at McLean Hospital that is studying whether Cymbalta duloxetine is neuroprotective in PD. The team's preliminary studies in mice showed that Cymbalta increased the expression of two proteins-engrailed homeobox 1 (En1) and forkhead box A2 (Foxa2) - that are necessary for dopaminergic neuron survival, "thus raising the possibility that the drug could prevent degeneration of those neurons in PD," Herpich said.

"Cymbalta is already marketed for other diseases, it is well tolerated in the elderly for long-term use" and a 2007 study by a group at Beilinson Hospital showed that the drug could treat pain in patients with PD, ${ }^{1}$ he said.

Eli Lilly and Co. markets Cymbalta, a selective serotonin and norepinephrine reuptake inhibitor (SSNRI), to treat depression, anxiety, pain, diabetic neuropathy, fibromyalgia syndrome and incontinence.

Another grant went to a team at the University of California, Los
Angeles that is exploring whether the generic TB vaccine bacillus Calmette-Guérin (BCG) can treat PD. That team "has demonstrated neuroprotective effects of the vaccine in a mouse model of PD," Herpich said. "Our research staff liked this one because it was 'out of the box' thinking and because the vaccine is safe, inexpensive and widely used" to prevent TB infection.

Herpich said the UCLA team's planned studies include elucidating the vaccine's mechanism of action in PD. He acknowledged that it was "tough to speculate what company might develop the vaccine to treat $\mathrm{PD}$, but perhaps the team's results would lead to follow-on funding" from MJFF to further advance its development.

According to a July 28 press release from MJFF, the remaining four grants went to an NIH team, an academic team in Michigan and two drug companies.

A team from the National Institute on Aging and the National Institute on Drug Abuse is conducting preclinical studies of four dipeptidyl peptidase-4 (DPP-4) inhibitors as neuroprotectants in animal models of PD. The inhibitors include three undisclosed drugs marketed to treat type 2 diabetes and Pfizer Inc.'s PF-00734200, which has completed Phase II testing to treat diabetes and renal disease.

A team at the Van Andel Institute and Michigan State University is investigating whether the rho kinase inhibitor fasudil could increase neuronal regeneration in the brains of animal models of PD. Asahi Kasei Pharma Corp. markets Eril fasudil to treat aneurysm.

Melior Discovery Inc. is testing the potential of MLR-1017-an undisclosed drug marketed to treat attention deficit hyperactivity disorder (ADHD) - to promote dopamine production in animal models of PD.

NeuroHealing Pharmaceuticals Inc. has NH004, an intraoral mucoadhesive dissolving film for the local delivery of tropicamide, in Phase II testing to treat sialorrhea (uncontrolled drooling) in PD patients. The company began the trial in 2008 with a 2007 grant under MJFF's clinical intervention program. Tropicamide is a topical, generic anticholinergic used to dilate the pupils during ophthalmological examination.

Herpich said that by this fall the foundation expects to announce a seventh repurposing grant for an undisclosed clinical project. $\mathrm{He}$ added that although the foundation has supported drug repurposing projects in the past, the new grant program was the first to solicit repurposing-specific applications from researchers.

\section{Early risk reduction}

In contrast to its new repurposing program, MJFF has historically focused primarily on understanding PD biology and advancing the development of new therapies for the disease.

Each year, the foundation allocates $\$ 2$ million to early discovery projects such as target validation and understanding the biology of a particular target or pathway. 
For example, in 2005 and 2007 two foundation grants worth $\$ 173,000$ and $\$ 267,000$, respectively, enabled a team at Lund University to show that inhibiting metabotropic glutamate receptor subtype 5 (mGluR5; GRM5) could treat levodopa-induced dyskinesia in animal models of PD.

Scherer noted that Addex Pharmaceuticals Ltd. and Novartis AG are now developing mGlu5R antagonists for PD, "and in its published work Novartis refers back to the Lund validation studies as a rationale for why the company decided to explore this target."

Additionally, through its Therapeutic Development Initiative (TDI), MJFF grants $\$ 5$ million per year exclusively to biopharma companies to fund lead optimization, pharmacokinetics, pharmacodynamics, dosing and early proof of concept studies in animals.

A case in point is the foundation's $\$ 300,000$ grant to reMYND N.V. to conduct preclinical dose-response studies of its lead PD therapeutic, ReS9-S7, a small molecule against an undisclosed target that inhibits neurotoxicity associated with $\alpha$-synuclein (SNCA) aggregation.

According to reMYND CSO Gerard Griffioen, by 2009 "we knew we had a promising lead compound in ReS9-S7, but we needed more data to move the program along to the clinic. Specifically, we needed preclinical dose-response data to estimate the therapeutic dose. This was a crucial piece of information. A wide safety window is very important for a lifelong therapy."

The preclinical data package reMYND submitted to MJFF showed the biotech was "fairly advanced in their $\alpha$-synuclein-based therapeutic approach, and the protein is a high-priority target for MJFF because of its disease-modifying potential," said Mark Frasier, MJFF's director of research programs. "reMYND had gathered a lot of preliminary data, so they were really asking for support to put critical finishing touches on work they had already done."

The grant helped reMYND show that ReS9-S7 was active at very low doses in animals, "and this translated into a low predicted therapeutic dose and thus a large safety window in patients," Griffioen said. "This told us we had a high promise of success in the clinic."

With these data in hand, reMYND announced a deal with Roche in 2010 that encompassed the biotech's PD and Alzheimer disease (AD) programs. reMYND received an undisclosed upfront payment and is eligible for $€ 500$ million (about $\$ 723$ million) in milestones plus royalties. $^{2}$

"If our results for ReS9-S7 had indicated the need for a high therapeutic dose, then for sure it would have been more difficult for us to engage in the collaboration with Roche," Griffioen said. "So the support of The Michael J. Fox Foundation helped make this strategic alliance possible."

The foundation made two TDI grants in 2010 worth a total of $\$ 295,000$ to Signum Biosciences Inc. to develop compounds that promote the dephosphorylation of SNCA, thereby preventing the protein's hyperphosphorylation and consequent toxic aggregation that underlies PD.,4

Lastly, MJFF grants $\$ 3$ million annually to support Phase I to Phase IIa clinical trials, Sherer said.

In 2010, MJFF granted Addex Pharmaceuticals $\$ 900,000$ to fund a Phase IIa trial of dipraglurant-IR (ADX48621) to treat levodopa-induced dyskinesia in PD patients, he said. Dipraglurant-IR is an immediate release formulation of dipraglurant, a negative allosteric modulator of mGluR5.

\section{Tools for trade}

In addition to programs that promote the development of new PD therapies, MJFF is trying to lower the cost of PD research by funding the development of tools.

"For instance in 2010 we launched the Parkinson's Progression Marker Initiative, a five-year, $\$ 45$ million program to identify biomarkers for PD," Sherer said. The program includes about 150 patients in the U.S. and EU, and the foundation plans to expand into other countries this year.

"As data are collected they are being made available online to accredited researchers," he noted. This information is available at the Parkinson's Progression Marker Initiative website (http://www.ppmi-info.org/).

MJFF develops other tools - such as antibodies and animal models - and makes those available to industry and academic researchers without licensing fees, though material transfer agreements are sometimes required, Herpich said.

The foundation funded the development of antibodies against leucine-rich repeat kinase 2 (LRRK2) and made those available to researchers on the sole condition that the researchers shared their results with MJFF. "So far over 150 researchers have done so," Sherer said.

This month, MJFF announced a collaboration with researchers at The University of British Columbia and Elan Corp. plc to develop a new mouse model of PD that expresses mutant mouse vacuolar protein sorting 35 homolog (Vps35). The British Columbia researchers led a multinational team that reported the mutant protein's role in PD in The American Journal of Human Genetics. ${ }^{5}$

Haas, M.J. SciBX 4(29); doi:10.1038/scibx.2011.813 Published online July 28, 2011

\section{REFERENCES}

1. Djaldetti, R. et al. Clin. Neuropharmacol. 30, 201-205 (2007)

2. Hansen, S. BioCentury 18(41), A6-A7; Sept. 20, 2010

3. Lee, K.-W. et al. J. Neurosci. 31, 6963-6971 (2011)

4. Haas, M.J. SciBX 4(21); doi:10.1038/scibx.2011.590

5. Vilariño-Güell, C. et al. Am. J. Hum. Genet. 89, 162-167 (2011)

COMPANIES AND INSTITUTIONS MENTIONED

Addex Pharmaceuticals Ltd. (SIX:ADXN), Geneva, Switzerland

Asahi Kasei Pharma Corp., Tokyo, Japan

Beilinson Hospital, Petah Tikva, Israel

Elan Corp. plc (NYSE:ELN), Dublin, Ireland

Eli Lilly and Co. (NYSE:LLY), Indianapolis, Ind.

Lund University, Lund, Sweden

McLean Hospital, Belmont, Mass.

Melior Discovery Inc., Exton, Pa.

The Michael J. Fox Foundation for Parkinson's Research,

New York, N.Y.

Michigan State University, East Lansing, Mich.

National Institute on Aging, Bethesda, Md.

National Institute on Drug Abuse, Bethesda, Md.

National Institutes of Health, Bethesda, Md.

NeuroHealing Pharmaceuticals Inc., Newton, Mass.

Novartis AG (NYSE:NVS; SIX:NOVN), Basel, Switzerland

Pfizer Inc. (NYSE:PFE), New York, N.Y.

reMYND N.V., Leuven, Belgium

Roche (SIX:ROG; OTCQX:RHHBY), Basel, Switzerland

Signum Biosciences Inc., Monmouth Junction, N.J.

The University of British Columbia, Vancouver,

British Columbia, Canada

University of California, Los Angeles, Calif.

Van Andel Institute, Grand Rapids, Mich. 